\title{
LA VACUNA CONTRA EL VIRUS DEL PAPILOMA HUMANO: ESTADO DE LA CUESTIÓN, PRINCIPIO DE PROPORCIONALIDAD Y CONSENTIMIENTO INFORMADO
}

\begin{abstract}
Boris Julián Pinto Bustamante ${ }^{1}$
Resumen: Entre los debates actuales en torno a las prácticas de vacunación, la vacuna contra el Virus de Papiloma Humano (VPH) formula diversos desafíos desde la bioética: por una parte, existen controversias en cuanto al perfil de eficacia y seguridad de las vacunas comercializadas, así como respecto de su costo-efectividad. Es evidente en estudios empíricos que el proceso de consentimiento informado no ofrece los elementos necesarios para que las pacientes y sus representantes legales puedan participar de forma significativa en el proceso de toma de decisiones en torno a la vacunación. El presente artículo presenta una revisión sobre el estado de la cuestión, ofrece un análisis desde la bioética a partir del principio de proporcionalidad y el método deliberativo-sincrético y sugiere algunos aportes para optimizar el proceso de consentimiento informado para la vacuna contra el VPH.
\end{abstract}

Palabras clave: vacunación, programas de inmunización, vacunas contra papillomavirus, bioética, consentimiento informado

The vaccine against Human Papilloma Virus: state of the question, proportionality principle and informed consent

\begin{abstract}
Within the current discussions on immunization practices, Human Papillomavirus (HPV) made various challenges from bioethics: firstly, there are controversies regarding the efficacy and safety profile of marketed vaccines, and with respect to their cost-effectiveness. Around the discussion is evident in empirical studies that the informed consent process does not provide the necessary elements for the patients and their legal representatives can participate meaningfully in the process of making decisions about vaccination. This article presents an overview of the status of the issue, with an analysis from bioethics from the principle of proportionality and the deliberative syncretic method and suggests some input to optimize the process of informed consent for the HPV vaccine.
\end{abstract}

Key words: vaccination, immunization programs, papillomavirus vaccines, bioethics, informed consent

$$
\begin{gathered}
\text { A vacina contra o Virus do Papiloma Humano: situação da questão, princípio de proporcionalidade e consentimento } \\
\text { informado }
\end{gathered}
$$

Resumo: Entre os debates atuais em torno das práticas de vacinação, a vacina contra o vírus do Papiloma Humano (HPV) formula diversos desafios a partir da bioética: por uma parte, existem controvérsias quanto ao perfil de eficácia e segurança das vacinas comercializadas, assim como a respeito de seu custo-efetividade. É evidente em estudos empíricos que o processo de consentimento informado não oferece os elementos necessários para que as pacientes e seus representantes legais possam participar de forma significativa no processo de tomada de decisóes em torno da vacinação. O presente artigo apresenta uma revisão sobre a situação da questão, oferece uma análise a partir da bioética, tendo como base o princípio da proporcionalidade e o método deliberativo-sincrético e sugere algumas contribuiçóes para otimizar o processo de consentimento informado para a vacina contra $\mathrm{OHPV}$

Palavras-chave: vacinação, programas de imunização, vacinas contra papillomavirus, bioética, consentimento informado

\footnotetext{
${ }^{1}$ Departamento de Bioética, Universidad El Bosque, Bogotá, Colombia Correspondencia: borisjpinto@gmail.com
} 


\section{Introducción}

Desde su introducción, la vacuna para la prevención de la infección por Virus del Papiloma Humano (VPH) ha suscitado controversias, inclinando la percepción pública entre la esperanza y la incertidumbre(1), particularmente respecto de su promoción como estrategia principal en la prevención del cáncer cérvico uterino (CCU), lo cual se traduce en resistencias de algunos sectores a las iniciativas de vacunación, objeciones de algunos profesionales de salud, multiplicación de la polémica en los medios masivos de comunicación(2), heterogeneidad en los conocimientos de los médicos ginecólogos sobre la infección por VPH y la vacunación $(3,4)$ y entre la población general(5), y divergencias en la información que se suministra a los padres y pacientes en el proceso de consentimiento informado(CI).

El propósito de este artículo de revisión es explorar el estado de la cuestión sobre el tema de la vacunación para la prevención de la infección por VPH y su relación con la práctica del CI que se ofrece a los padres y pacientes a partir de algunas premisas: la etiopatogenia de la infección por VPH y la inmunidad natural en relación con el $\mathrm{CCU}$, la controversia sobre la efectividad y la seguridad de las vacunas comercializadas a la fecha, las consideraciones sobre la vacuna contra el VPH en el contexto de la salud pública en términos de costo-efectividad, las dificultades y desafíos en la práctica del CI sobre el tema en cuestión y el análisis ético a la luz del principio de proporcionalidad y el modelo deliberativo-sincrético propuesto por Diego Gracia.

\section{Resultados}

Inmunidad natural y etiopatogenia de la infección por VPH

Algunos autores consideran que la inmunidad ofrecida por las vacunas actualmente disponibles contra el VPH no es claramente superior a la inmunidad natural. La infección por VPH es la infección de transmisión sexual más prevalente. Alrededor del $80 \%$ de mujeres adquirirán la infección en el curso de su vida(6). Sin embargo, no todas desarrollarán alguna forma de cáncer relacionado con la infección. De los diferentes sub- tipos de VPH, alrededor de 15 subtipos son responsables del CCU(7), si bien parece claro que la presencia de la infección por VPH es un elemento necesario pero no suficiente para el desarrollo de displasias y lesiones cancerosas(8).

La infección por los subtipos 16 y 18 de VPH no parece ser la "causa central e independiente" del CCU(9:2-3). Aunque existe una distribución similar de los genotipos 16 y 18 de VPH entre países industrializados y países en vías de desarrollo, las tasas de enfermedad, la incidencia, la prevalencia y la mortalidad por CCU son claramente más altas en países en vías de desarrollo(9:4). De allí que no se pueda deducir que la infección por los genotipos 16 y 18 sea el único factor implicado en la génesis del CCU.

El 90\% de las infecciones por VPH exhiben un curso benigno, subclínico y autolimitado(8), y solo un $1 \%$ de las infecciones por VPH progresa a CCU(10). La infección por VPH de alto riesgo (particularmente los subtipos 16 y 18, cuya prevalencia en Estados Unidos es de alrededor del 2,3\%)(11) tiene una duración media de entre 12 y 18 meses(12) y hasta 24 meses según otros reportes(8). En la mayoría de los casos la infección, incluso por subtipos carcinógenos, suele ser eliminada por el sistema inmunológico(6), y un número importante de infecciones asociadas con displasias de bajo grado tiende a regresar de forma espontánea. Alrededor del 70\% de las infecciones expresadas en cambios en la citología cervicovaginal (CCV) serán eliminadas en el curso de un año y el $90 \%$ en el curso de tres años. El 20\% de las neoplasias cervicales intraepiteliales (NIE) tardarán alrededor de cinco años en progresar hasta cáncer invasivo $\mathrm{y}$, tras treinta años a partir de la infección, solo el $40 \%$ habrá alcanzado tal estado de desdiferenciación celular(10).

Aproximadamente un $10 \%$ de las mujeres desarrolla un mecanismo de persistencia a largo plazo tras la infección por VPH de alto riesgo(12). De este porcentaje, cerca de la mitad evoluciona hasta NIE precancerosa grado 2 ó 3. Este mecanismo de persistencia favorece la aparición de verrugas anogenitales, lesiones precancerosas y cáncer de tracto anogenital y de orofaringe(6). Además del mecanismo de persistencia, otros factores intervienen en la posibilidad de desarrollar displasias 
de alto grado o lesiones cancerosas: el uso de anticonceptivos orales, la edad de inicio de relaciones sexuales, la paridad, el tabaquismo, deficiencias nutricionales, coinfecciones (Chlamydia Trachomatis), el no uso consistente de preservativo y las conductas sexuales de riesgo(8), como es el caso de mujeres que han tenido tres o más compañeros sexuales en los últimos 12 meses, si bien parece claro que cualquier mujer sexualmente activa está en riesgo de contraer la infección(13).

La controversia en torno a la efectividad y seguridad de las vacunas para la prevención de la infección por VPH

Los estudios en fase III, FUTURE (I y II) y PATRICIA, de las primeras vacunas comercializadas (la vacuna tetravalente HPV-6/11/16/18 - Gardasil; la vacuna bivalente HPV-16/18 - Cervárix, respectivamente) ofrecen una efectividad estimada para la prevención de NIE grado 2 y 3 relacionadas con subtipos 16 y 18 que oscila entre el $90,4 \%$ hasta el 98\%, mientras que la eficacia global de la vacuna contra la aparición de NIE varía entre el $89,2 \%$ y el $100 \%$ respectivamente(6). Las dos vacunas sugieren efectividad en la prevención de condilomas, adenocarcinoma in situ, neoplasia intraepitelial vaginal, neoplasia intraepitelial anal, asociados con subtipos oncogénicos de VPH, aunque subsiste la controversia frente al periodo de efectividad real en la prevención de NIE grado 2 y 3, así como de CCU invasivo (9,4 años para la vacuna bivalente, 5 años para la vacuna tetravalente en ensayos clínicos controlados(14), lo cual indicaría que ambas vacunas se encuentran por debajo del umbral necesario para la prevención de CCU, el cual ha sido estimado en 15 años(14) (con al menos 90\% de eficacia demostrada contra VPH 16, para que se considere una estrategia costo-efectiva)(15). Otros informes sugieren una duración del efecto protector de hasta treinta años o la necesidad de refuerzos periódicos(16), de lo que, si la duración de la protección es inferior a treinta ańos y la efectividad es del $70 \%$, su costo-efectividad sería nula(17).

Otra de las objeciones sugiere que el criterio indirecto establecido por el comité consultor de la OMS para la determinación de la efectividad de la vacuna para el VPH (NIE de grado moderado o severo - 2/3 o CCU)(18), en uno de los principales estudios en el desarrollo de la vacuna tetravalente (FUTURE II), es un criterio irreal, dado que en la población estudiada (mujeres entre los 15 y los 26 años de edad), y teniendo en cuenta el periodo de latencia de la infección por $\mathrm{VPH}$, las lesiones precancerosas o el CCU no son condiciones prevalentes(19). Por otra parte, la utilización de las NIE grado 2 puede no ser el mejor marcador para definir la efectividad de las vacunas, pues un alto porcentaje de estas lesiones resuelven espontáneamente, independientemente de la vacunación (en mujeres entre 13 y 24 años, $38 \%$ antes de un año, $63 \%$ después de dos años, $68 \%$ después de tres años) y es, además, un diagnóstico histopatológico que no ofrece la mejor reproducibilidad diagnóstica, tanto por errores en la obtención de las muestras, como por la alta variabilidad entre observadores(20).

Diversos estudios sugieren un adecuado perfil de seguridad de las vacunas comercializadas en la actualidad, tanto para la vacuna tetravalente(21) como para la bivalente, en la mayoría de mujeres de diversos rangos de edad y hasta los 72 años (10). El Sistema de Reporte de Eventos Adversos por Vacunas (Vaccine Adverse Events Reporting System, VAERS), tras 18 meses de la comercialización de la vacuna tetravalente en Estados Unidos, registró algunos efectos colaterales como síncope $(8,2$ casos por 100.000 dosis distribuidas), tromboembolismo venoso (0,2 casos por 100.000 dosis) e informes que incluyen desde reacciones alérgicas, hasta pancreatitis y síndrome de Guillain-Barré (3,4 casos por 100.000 dosis).

Los informes de casos por parte de investigadores independientes dan cuenta de estos y otros posibles efectos adversos relacionados con la vacuna tetravalente contra VPH. Si bien los informes aislados de casos no constituyen la mejor evidencia en cuanto a la seguridad de la vacuna, su importancia no se puede subestimar y son necesarios más estudios poscomercialización y de farmacovigilancia que permitan establecer nexos reales de causalidad entre los eventos reportados y la aplicación de la vacuna(14). Se han informado casos de síndrome ASIA (síndrome autoinmune/ inflamatorio inducido por adyuvante) relacionados con la aplicación de la vacuna contra el VPH, así como cuadros de disautonomía, actualmente en investigación. Estos informes no deben deses- 
timarse y demandan, por el contrario, un seguimiento exhaustivo y personalizado siguiendo el principio de precaución(21).

\section{Vacunas contra el VPH, costo-efectividad y sa- lud pública}

El sustento para justificar la inmunización masiva contra el VPH depende de varias premisas: solo el 50\% de las mujeres infectadas con subtipos oncogénicos desarrollan anticuerpos contra la infección por VPH. La respuesta inmune celular natural es lenta, débil y no confiere inmunidad específica ante nuevas infecciones(6). El CCU es el segundo tipo de cáncer más prevalente en el mundo, con cerca de 450.000 casos nuevos diagnosticados cada año(12), con una tasa de incidencia que oscila entre 1-50 por cada 100.000 mujeres. Las mismas tasas, al igual que los índices de mortalidad, son mayores en países en vías de desarrollo(23). La infección por VPH está relacionada con otras formas relativamente raras de cáncer como son: vagina, vulva, pene (entre 40-60\%, relacionados con infección por $\mathrm{VPH})$, ano $(80 \%$ relacionado con la infección por VPH), algunos tipos de cáncer de cabeza y cuello, así como en la aparición de verrugas anogenitales, neoplasias de bajo grado y papilomatosis respiratoria recurrente(23). En este sentido, la vacuna puede contribuir en la prevención de otras formas de cáncer y las evidencias sugeridas de protección cruzada frente a distintos subtipos respaldarían esta indicación(24).

Algunos estudios sugieren que las prácticas de inmunización, acompañadas de un aumento en las coberturas de cribado, son iniciativas que pueden contribuir a optimizar la equidad en salud, particularmente para poblaciones en situación de riesgo(25). Un informe sugiere que, cuatro años después de la introducción de la vacuna tetravalente, se registran evidencias que respaldan una disminución en la prevalencia de infección por subtipos de VPH relacionados con la vacuna, incluso entre mujeres jóvenes no vacunadas, lo cual sugiere una inmunidad de rebaño en ciertas comunidades(26).

La vacuna bivalente ofrece una reducción en la aparición de casos de NIE grados 2 y 3 causadas por cinco serotipos diferentes de VPH, así como la posibilidad de prevenir una de cinco CCV anormales por infección por VPH y alrededor del $70 \%$ de tratamientos excisionales en mujeres con acceso a cribado, con una reducción de hasta el $90 \%$ en la incidencia de adenocarcinomas en poblaciones con acceso a cribado(10). La vacuna tetravalente ofrece un perfil similar en cuanto a la reducción de NIE grados 2 y 3 a mujeres que acceden a estrategias de cribado, así como un $10 \%$ en reducción de citologías normales, un $20 \%$ en la disminución de colposcopias y un $42 \%$ en la realización de tratamientos. Pero la incidencia de CCU entre mujeres con acceso a CCV, más aplicación de vacuna tetravalente, no es menor que la incidencia en mujeres con acceso a CCV sin vacunación(14).

Tanto para la vacuna tetravalente, como para la vacuna bivalente, los datos recabados hasta el momento señalan que el principal propósito de la vacunación, la prevención del CCU, se puede alcanzar con mayor eficacia por medio de los programas de cribado mediante la CCV, con la vacunación como una opción en países con dificultades en el acceso a CCV(10). La estrategia de prevención secundaria mediante cribado es más costo-efectiva que la aplicación de la vacuna y que el cribado más la inmunización(14).

Un estudio realizado en Colombia sugiere que, aunque la vacunación contra VPH puede ser una alternativa viable para la prevención del CCU en el país, los precios de cada vacuna deberían disminuir entre un 32\% a un 36\% para convertir la vacunación en una estrategia costo-efectiva en el contexto local(27). La evaluación de costo-efectividad de las vacunas para el VPH depende al tiempo de dos factores fundamentales: la sensibilidad y la cobertura de la CCV. A menor sensibilidad de la prueba de cribado y menor cobertura, mayor costo-efectividad de la vacunación, de lo cual se puede deducir que si se optimizan la calidad de la prueba de cribado y las estrategias de cobertura para garantizar el acceso oportuno de las mujeres a la CCV, se incrementa la costoefectividad de esta última respecto de la estrategia de vacunación.

Una pregunta similar en relación con el precio de las vacunas y la costo-efectividad de los programas de vacunación para VPH plantea otro informe en 
la India(28). Un informe en Australia afirma que, dado que las mujeres inmunizadas deben seguir realizando su CCV periódica, no son costo-efectivos los programas de vacunación contra VPH, particularmente en países donde la CCV y la cirugía han disminuido las tasas de mortalidad por $\operatorname{CCU}(9)$.

En diversos países se ha incluido la prueba de ADN VPH en los planes de beneficios. Un estudio sobre costo-efectividad de esta prueba de prevención secundaria, en un país como Colombia (donde fue incluida la prueba en el Plan Obligatorio de Salud), sugiere que la tamización con prueba ADN VPH es costo-efectiva, particularmente si se optimizan niveles de cobertura y tasas de seguimiento(29). Otro estudio que compara la CCV con la combinación de la CCV más la prueba ADN VPH, y la CCV más la vacunación, sugiere que la CCV sigue siendo la estrategia más costo-efectiva, seguida por la prueba ADN VPH en combinación con la CCV trianual. El estudio afirma que la combinación de CCV trianual asociada a vacunación puede ser costo-efectiva si se reducen a gran escala los costos por vacunación(30). Otro informe sobre costo-efectividad en Tailandia concluye que los costos de la vacuna por dosis aplicada en preadolescentes deben disminuir para que esta estrategia, sumada a la CCV realizada al menos cinco veces en el curso de la vida, sea costo-efectiva. Sugiere también que si la vacunación no es factible, la prueba de ADN VPH es eficiente(31).

En Chile, un informe técnico de revisión y análisis de estudios de costo-efectividad en 2011 no recomendó la incorporación de la vacuna contra el VPH(32). Sin embargo, a pesar de los informes técnicos y del descenso en la mortalidad asociada al CCU en ese país en los últimos años, gracias a la CCV, el Ministerio de Salud chileno incorporó la vacuna en el plan nacional de inmunización en 2014(33). Mientras se generaliza la vacunación contra el VPH, bajo la presunción de efectividad de por vida, aún no demostrada empíricamente, ya se evidencia un retroceso importante en la cobertura de cribado en ese país, lo cual puede comprometer los resultados en salud púbica a mediano y largo plazo(33).

\section{Consentimiento informado y vacunación contra el VPH}

Diversas inconsistencias debilitan la validez del proceso de CI y lesionan la confianza pública sobre los beneficios reales de la vacunación contra el VPH(34): la polémica suscitada por posibles conflictos de interés alrededor de las iniciativas de vacunación contra el VPH(35), la controversia acerca de la vulneración de principios éticos en la conducción de algunos ensayos clínicos en países como la India(36), el intenso cabildeo promovido por sectores de la industria farmacéutica para impulsar la comercialización de la vacuna y la recomendación de la vacunación obligatoria, incluso a través de la financiación de grupos de presión(37), las controversias en torno a la eficacia a largo plazo de la vacuna cuadrivalente en la prevención de la neoplasia intraepitelial y el cáncer anal(38), las inconsistencias de algunos estudios (como la afirmación prematura sobre la relación entre disminución de la prevalencia de infección por VPH y la aplicación de una dosis de vacuna tetravalente, atribuyendo a la aplicación de la vacuna un resultado que puede deberse a cambios en el comportamiento sexual)(39), los conflictos de interés que dificultan la regulación de ensayos clínicos en países como Costa Rica(40), y las acusaciones por la violación de los códigos de publicidad de salud en Francia, donde se ofrecía una propaganda no ajustada a la mejor evidencia científica disponible en el momento(41), han contribuido en la resistencia de algunos sectores frente a la vacunación contra el VPH.

Un estudio realizado en Canadá encontró imprecisiones y discrepancias entre la información suministrada entre distintos Estados en el proceso de CI, particularmente en lo referente a los riesgos físicos de la vacunación(33). Otro informe en Ontario informó que, aunque todos los centros de salud solicitaron CI, existen discrepancias respecto del CI por sustitución otorgado por los padres(42). Algunos autores proponen que los adolescentes mayores de 12 años (en Estados como Texas y California en los Estados Unidos) puedan otorgar su CI sin el concurso de sus representantes legales(43).

Un estudio cualitativo encontró que, a pesar de ser altamente ofrecida la vacuna del VPH en Es- 
tados Unidos, es muy baja la tasa de vacunación. En este estudio se encontró que las adolescentes se consideran a sí mismas como participantes pasivas en los procesos de toma decisión frente a la aplicación de la vacuna, siendo sus madres quienes generalmente toman la decisión final(44).

$\mathrm{El}$ arte del CI consiste en garantizar una traducción veraz, actualizada y transparente sobre los aspectos críticos y necesarios para que el paciente y/o su representante legal puedan tomar una decisión autónoma(45). Demanda, a su vez, un intérprete que facilite ese proceso de comunicación, que actúe de forma independiente y que tenga la capacidad para evaluar de forma crítica la información científica disponible(46).

Dado el nivel actual de incertidumbre sobre el tema, y por tratarse de un punto sensible para la salud pública, no es razonable dejar la confección del CI a la discrecionalidad de cada institución de salud. Se debe promover desde las autoridades regulatorias locales y los ministerios de salud, asesorados por grupos de expertos independientes, la elaboración de guías sobre el proceso de CI, así como la generación de espacios pedagógicos para los profesionales de salud y la comunidad.

Algunos aspectos fundamentales que debe considerar el CI para la vacuna contra el VPH, transcritos y explicados en un lenguaje comprensible, con el acompañamiento del profesional de salud y desde una perspectiva relacional y deliberativa que promueva la autonomía de las partes involucradas (padres, niños/niñas, profesionales de salud)(47), pueden ser:

- Naturaleza de la infección por el VPH (medio de trasmisión, tipos de VPH, etc.).

- Relación entre la infección por virus VPH y la aparición de lesiones precancerosas y CCU (como factor necesario más no suficiente).

- Cofactores asociados a la infección por VPH en la génesis del CCU.

- Relación de la infección por VPH y la aparición de otro tipo de condiciones (verrugas, condilomas, cáncer de ano, garganta, pene, etc.).
- Papel de la inmunidad natural en la infección por VPH.

- Evolución natural de la infección por VPH y porcentajes estimados de aparición de lesiones precancerosas y CCU en la población general.

- Estrategias de prevención primaria para la infección por VPH, diferentes a la vacunación (uso consistente de preservativo, prácticas sexuales seguras, incremento en la edad de inicio de la actividad sexual, abandono del consumo de tabaco, importancia de la circuncisión en la prevención de enfermedades de trasmisión sexual, baja paridad, uso de micronutrientes, uso controlado de métodos anticonceptivos orales basados en combinación de estrógenos/progestágenos), así como estrategias para la disminución de la persistencia, progresión y regresión de la infección, como la utilización de algunos suplementos vitamínicos y agentes quimioterapéuticos presentes en la dieta (Naringin, indoles, etc.) (48).

- Estrategias de prevención secundaria para la infección por VPH (énfasis en la importancia de la práctica periódica de la CCV, aun tras la administración del esquema de vacunación contra el VPH). Aclarar que la prevención del CCU se puede lograr por otros medios diferentes a la vacunación(49).

- Composición de la vacuna, tipo de inmunidad que ofrece (producción de anticuerpos, sin modificación de la inmunidad celular).

- Esquema de administración.

- Efectividad real de la vacuna (a la luz de la mejor evidencia disponible). Aclaración de que, a la fecha, ninguna vacuna contra el VPH puede prevenir todas las formas de infección anogenital por este virus(50).

- Posibles efectos adversos (a la luz de la mejor evidencia disponible).

Si existe incertidumbre científica acerca de los puntos anteriores, tal información debe ser suministrada de forma transparente durante el proceso de consentimiento. La información proporciona- 
da debe actualizarse de acuerdo con los resultados relevantes en investigación. Se puede proponer un CI con una entrevista de apoyo, articulada a partir de preguntas tipo cuestionario, que permitan indagar el conocimiento real del/la paciente y su representante legal y resolver las inquietudes durante el proceso(5).

\section{Discusión}

A pesar de los esfuerzos por convertir la medicina en una ciencia que aspira a la certeza, toda decisión clínica siempre se toma en escenarios de incertidumbre, por lo cual cada decisión médica requiere de prudencia. En tal sentido, Diego Gracia recuerda la prudencia aristotélica como recta radio agibilum(51:99), de donde la recta radio se corresponde con un primer momento deontológico y universal, mientras que agere corresponde a un segundo momento práctico y teleológico. Así, el axioma (norma universal y general) y la máxima retórica (como "como criterios sabios y prudentes de actuación práctica”)(51:102) no se contradicen, sino, mejor, se complementan en el ejercicio práctico de la prudencia. Si el primer momento, deontológico y universal, exige el cumplimiento de las obligaciones morales como imperativos categóricos, el segundo momento, teleológico o de las consecuencias prácticas, permite identificar y justificar las excepciones al axioma.

La medicina, la ética y la política son saberes provisionales, contingentes e históricos, más que ciencias que aspiren al saber universal y absoluto(51:9). En este escenario de perplejidad se mueve también la investigación biomédica que, en el caso de la vacuna contra la infección por VPH, ofrece un margen de incertidumbre que excede las expectativas generadas en la opinión pública.

Aunque algunos reportes de eventos adversos sugieren un aceptable perfil de seguridad en términos estadísticos (no maleficencia), la incertidumbre acerca de la efectividad de la vacuna a largo plazo (beneficencia) no justificaría exponer a grandes poblaciones a efectos adversos sin potencial clínico evidente en el momento de su aplicación.

El principio de proporcionalidad propone un equilibrio justo y razonable entre riesgos, cargas y potenciales beneficios, entre beneficencia y no maleficencia, entre costos y efectividad, tanto individual como socialmente, así como entre distintos intereses relevantes (de la ciencia, del investigador, del individuo, comerciales, de las personas presentes y futuras, públicos y de la sociedad, entre otros) por medio de un ejercicio de ponderación y priorización(52:45-51).

En el caso de una intervención ya aprobada, si hay beneficios objetivos, es tolerable un umbral de riesgo previsto mínimo/aceptable ante una intervención médica o profiláctica (aceptación que debe ser ratificada por el paciente mediante un adecuado proceso de CI). Si no hay beneficios claramente superiores en comparación con otras estrategias de prevención, un evento adverso grave, en el contexto terapéutico y de la salud pública, puede ser inadmisible. Un caso que refleja esta afirmación es el caso del ensayo clínico para el desarrollo de una vacuna contra el meningococo B en Noruega en los años ochen$\operatorname{ta}(52: 149,165,166)$. En este escenario, la baja tasa de complicaciones (4.3 por cada 100.000 dosis administradas) es desproporcionada respecto del exiguo margen de beneficios reales (efectividad de la vacuna del 57,2\%). El caso señalado era un ensayo clínico, a diferencia de las estrategias de inmunización contra VPH ya comercializadas en muchos países.

Desde la perspectiva deontológica (o de los axiomas), siguiendo el principio de proporcionalidad, en cuanto es mayor el umbral de incertidumbre sobre los beneficios reales de una intervención médica, es menor el umbral aceptable de daño o riesgo(52), tanto en términos de cargas, riesgos físicos e individuales, como en términos de costo-efectividad y justicia distributiva, lo cual debe ratificarse en el proceso de CI como garantía del respeto por la autonomía de las personas. Desde la perspectiva teleológica (o de las máximas y consecuencias), siguiendo el principio de proporcionalidad, las divergencias en cuanto a la efectividad real de la vacuna contra el VPH y las dudas frente a su costo-efectividad no permiten justificar excepciones a las normas deontológicas (en este caso, los imperativos morales de no maleficencia y justicia distributiva).

Según los argumentos expuestos, algunos autores 
han propuesto una moratoria en la implementación de las iniciativas de vacunación contra el $\mathrm{VPH}(17)$ hasta tanto exista evidencia razonable que justifique, desde la consideración de las normas morales y las consecuencias prácticas, la razonabilidad de tal iniciativa. Si esto no es posible, dados los múltiples factores e intereses que gravitan en torno al problema, el punto crítico para la actuación prudente demanda, de una parte, el rol pedagógico de los profesionales de la salud en el proceso de CI, y, de otra, el papel de las agencias regulatorias locales en la priorización de iniciativas en salud pública que hayan demostrado eficacia, costo-efectividad y que se ajusten a la promoción de derechos humanos y principios éticos fundamentales, en contraposición con las iniciativas que no hayan demostrado estos atributos.

La vacuna ha demostrado protección contra la infección por algunos serotipos de $\mathrm{VPH}$, pero la mejor estrategia para la prevención del CCU, según la evidencia recabada hasta el momento, sigue siendo la CCV(47). La imposición o la aceptación pasiva de la vacuna contra el VPH puede ser un indicador de una menor participación futura en programas de cribado contra el CCU(49).

Aunque la vacuna contra el VPH ha sido incorporada como estrategia de prevención primaria en varios países(48), con unos índices positivos de aceptabilidad (como Argentina y Chile) (3,53), las divergencias en torno al conocimiento científico actual acerca de la efectividad, seguridad, inmunogenicidad y esquemas de administración de la vacuna(54) se reflejan en la diversidad de recomendaciones y de información que proveen los profesionales de salud, en la heterogeneidad de los formularios de CI y en los niveles de conocimiento entre el público general y los profesionales sanitarios.

\section{Conclusiones}

Siguiendo el método deliberativo-sincrético propuesto por Diego Gracia, que toma en consideración los momentos deontológico y teleológico en la evaluación de problemas éticos, y el principio de proporcionalidad, se concluye que, en el estado actual de la cuestión, no se justifica la amplia expectativa pública que se ha promovido en torno a la vacunación contra el VPH como estrategia de elección en la prevención del CCU.

Una información suficiente, consensuada y actualizada, según se conocen nuevos resultados de los estudios en curso, que tome en consideración las posibilidades emergentes (vacunas de segunda generación contra VPH - como la vacuna nonavalente disponible desde 2014 y con un perfil de eficacia e inmunogenicidad similar a la vacuna tetravalente(55)—, frecuencias de distribución de los serotipos de VPH tras la inmunización masiva, efectos de la vacunación masculina en la salud pública) debe ser parte de un proceso integral de CI desde un enfoque relacional, deliberativo y con participación comunitaria. Hasta tanto no se demuestre el periodo real de inmunogenicidad de las vacunas comercializadas y sus beneficios sociales en términos de costo-efectividad, no se debería proponer un programa de vacunación universal obligatorio financiado con fondos públicos, dado el impacto económico para la salud pública y las divergencias científicas enunciadas. En México, por ejemplo, se propuso la implementación de una política focalizada en los programas de vacunación contra $\mathrm{VPH}$, dirigidos a mujeres de estratos socioeconómicos bajos en poblaciones rurales y urbanas, con dificultades en el acceso a servicios de salud y a la CCV(56). No obstante, garantizar la vacunación sin mejorar el acceso a la CCV periódica y sin intervenir los determinantes sociales de la salud, sigue siendo una tarea incompleta.

En este punto, es fundamental apoyar la investigación independiente sobre el desarrollo de las vacunas contra el VPH y promover, desde las agencias regulatorias locales, la difusión de guías éticas y consensos sobre la confección de los documentos de $\mathrm{CI}(57)$, así como la generación de escenarios pedagógicos, tanto para los profesionales de la salud, como para la población general, conforme avanza la investigación sobre las vacunas contra el VPH. 


\section{Referencias}

1. Todorova I, Alexandrova-Karamanova A, Panayotova Y, Dimitrova E, Kotzeva T. Managing Uncertainty: Healthcare Professionals' Meanings Regarding the HPV Vaccine. Int J Behav Med 2013.

2. Eberth JM, Kline KN, Moskowitz DA, Montealegre JR, Scheurer ME. The Role of Media and the Internet on Vaccine Adverse Event Reporting: A Case Study of Human Papillomavirus Vaccination. J Adolesc Health 2013; 54(3): 289-295.

3. Mazzadi A, Paolino M, Arrossi S. Aceptabilidad y conocimientos sobre la vacunación contra el virus del papiloma humano (VPH) en médicos ginecólogos de la Argentina. Salud Pública Mex 2012: 54: 515-522.

4. Aldrich T, Becker D, García SG. Mexican physicians' knowledge and attitudes about the human papillomavirus and cervical cancer: a national survey. Sex Transm Infect 2005; 81: 135-141.

5. Somogyi L, Malpica CC, Carrillo D, Rodríguez E. Vacunación VPH. Propuesta bioética y consentimiento informado. Rev Obstet Ginecol Venez 2009; 69(1): 48-55.

6. Pandhi D \& Sonthalia S. Human papilloma virus vaccines: Current scenario. Indian Journal of Sexually Transmitted Diseases 2011; 32(2): 75-85.

7. Saslow D, Castle PE, Cox JT, Davey DD, Einstein MH, Ferris DG, Garcia F. American Cancer Society Guideline for human papillomavirus (HPV) vaccine use to prevent cervical cancer and its precursors. Cancer Journal for Clinicians 2007; 57(1): 7-28.

8. Tejeda D, Serrano M y Gómez-Pastrana F. Cáncer de cuello uterino. Estado actual de las vacunas frente al virus del papiloma humano (VPH). Oncología (Barc) 2007; 30(2): 19.

9. Wilyman J. HPV Vaccination programs have not been shown to be cost-effective in countries with comprehensive Pap screening and surgery. Wilyman Infectious Agents and Cancer 2013; 8(21): 2-4.

10. Harper D, Vierthaler S. Next Generation Cancer Protection: The Bivalent HPV Vaccine for Females. ISRN Obstetrics and Gynecology 2011.

11. Dunne EF, Unger ER, Sternberg M, McQuillan G, Swan DC, PatelSS \& Markowitz LE. Prevalence of HPV Infection among females in the United States. JAMA 2007; 297(8): 813-819.

12. Bodily J, Laiminis LA. Persistence of human papillomavirus infections: keys to malignant progression. Trends in Microbiology 2011; 19(1): 33-39.

13. Bahmanyar E, Paavonen J, Naud P, Salmerón J, Chow SN, Apter D, et al. Prevalence and risk factors for cervical HPV infection and abnormalities in young adult women at enrolment in the multinational PATRICIA trial. Gynecologic Oncology 2012; 127(3): 440-450.

14. Harper DM, Vierthaler SL, Santee JA. Review of Gardasil. J Vaccin Vaccinat 2010; 1(107): 1-7.

15. Heitmann E, Harper D. Prophylactic HPV Vaccines and the prevention of Cervical Intraepithelial Neoplasia (CIN). Curr Obstet Gynecol Rep 2012; 1(3): 95-105.

16. Brisson M, Van de Velde N, Boily MC: Economic evaluation of human papillomavirus vaccination in developed countries. Public Health Genomics 2009; 12: 343-351.

17. Gérvas J. La vacuna contra el virus del papiloma humano desde el punto de vista de la atención primaria en España. Rev Bras Epidemiol 2008; 11(3): 505-508.

18. Sonia R. Pagliusi, M. Aguado T. Efficacy and other milestones for human papillomavirus vaccine introduction. Vaccine 2004; 23: 577.

19. The Future II Study Group. Quadrivalent vaccine against human papillomavirus to prevent high-grade cervical lesions. New England Journal of Medicine 2007; 356(19): 1915-1927.

20. Tomljenovic L, Wilyman J, Vanamee E, Bark T, Shaw C. HPV vaccines and cancer prevention, science versus activism. Infectious Agents and Cancer 2013; 8(1): 6.

21. Lu B, Kumar A, Castellsagué X, Giuliano A. Efficacy and Safety of Prophylactic Vaccines against Cervical HPV Infection and Diseases among Women: A Systematic Review \& Meta-Analysis. BMC Infectious Disease 2011; 11(13): 1-16.

22. Anaya JM, Reyes B, Perdomo-Arciniegas, Camacho-Rodríguez, AMB, Rojas-Villarraga A. Autoimmune/autoinflammatory Syndrome induced by adjuvants (ASIA) after quadrivalent human papillomavirus vaccination in Colombians: a call for personalised medicine. Clinical and experimental rheumatology 2015; 33(4): 545-548.

23. Human papillomavirus vaccines. WHO position paper. Wkly Epidemiol Rec 2009; 84(15): 118-131.

24. Wheeler CM, Castellsagué X, Garland SM, Szarewski A, Paavonen J, Naud P, et al. Cross-protective efficacy of HPV16/18 AS04-adjuvanted vaccine against cervical infection and precancer caused by non-vaccine oncogenic HPV types: 4 -year end-of-study analysis of the randomised, double-blind PATRICIA trial. The Lancet Oncology 2012; 13(1): 100110. 
La vacuna contra el virus del papiloma humano - Boris Julián Pinto Bustamante

25. Crowcroft NS, Hamid JS, Deeks SL, Frank J. Human papilloma virus vaccination programs reduce health inequity in most scenarios: a simulation Study. BMC Public Health 2012; 12(1): 935.

26. Kahn JA, Brown DR, Ding L, Widdice LE, Shew ML, Glynn S, et al. Vaccine-type human papillomavirus and evidence of herd protection after vaccine introduction. Pediatrics 2012; 130(2): 249-256.

27. Aponte J, Fajardo L, Diaz J, Eslava J, Gamboa O, et al. Cost-Effectiveness Analysis of the Bivalent and Quadrivalent Human Papillomavirus Vaccines from a Societal Perspective in Colombia. PLoS ONE 2013; 8(11): e80639.

28. Farhath S, Vijaya P, Mumtaj P. Cervical Cancer: Is Vaccination Necessary in India? Asian Pacific J Cancer Prev 2013; 14 (4): 2681-2684.

29. Andrés-Gamboa O, Chicaíza L, García-Molina M, et al. Cost-effectiveness of conventional cytology and HPV DNA testing for cervical cancer screening in Colombia. Salud Pública de México 2008; 50(4): 276-285.

30. Chen MK, Hung HF, Duffy S, Yen AM, Chen HH. Cost-effectiveness analysis for Pap smear screening and human papillomavirus DNA testing and vaccination. J Eval Clin Pract 2011; 17(6): 1050-1058.

31. Sharma M, Ortendahl J, Van der Ham E, Sy S, Kim JJ. Cost-effectiveness of human papillomavirus vaccination and cervical cancer screening in Thailand. BJOG 2012; 119(2): 166-176.

32. Castillo M, Castillo C, Aravena M. Sistematización de la Información sobre Cáncer Cérvico Uterino en Chile: Revisión y Análisis de Estudios de Costo-efectividad de la Vacuna contra VPH. Santiago de Chile: Ministerio de Salud, Departamento de Economía de la Salud División de Planificación Sanitaria Subsecretaría de Salud Pública; 2011.

33. Fica A. Prevención del cáncer cérvico-uterino en Chile. Mucha vacuna y poco Papanicolau. Rev Chilena Infectol 2014; 31(2): 196-203.

34. Steenbeek A, Macdonald N, Downie J, Appleton M \& Baylis F. Ill-informed consent? A content analysis of physical risk disclosure in school-based HPV vaccine programs. Public Health Nursing 2012; 29(1): 71-79.

35. Wolfe SM. Q\&A on the human papilloma virus vaccine Gardasil. Health Letter. (Public Citizen Health Research Group) 2007; 23: 1-3.

36. Sarojini NB, Srinivasan S, Madhavi Y, Srinivasan S, Shenoi A. The HPV vaccine: science, ethics and regulation. Econom Polit Weekly 2010; 45: 27-34.

37. Ugalde A, Homedes N. Medicamentos para lucrar. La transformación de la industria farmacéutica. Salud Colectiva 2009; 5(3): 305-322.

38. Talboy GE, Lankachandra K, Harper DM. Speculation overinflates long-term efficacy of vaccine for anal displasia. The Lancet Oncology 2013; 14(7): E249-E250.

39. Groner JA, Harris GD, Harper DM. Reduction in HPV prevalence no evidence to support HPV vaccination reduces HPV prevalence. J Infect Dis 2014; 209(8): 1302-1304

40. Homedes N, Ugalde A. Clinical Trials in Latin America: Where Ethics and Business Clash. Cham: Springer International Publishing Switzerland; 2014: 189-190.

41. Tomljenovic L, Shaw CA. Human papillomavirus (HPV) vaccine policy and evidence-based medicine: are they at odds? Ann Med 2013; 45(2): 182-193.

42. Wilson SE, Karas E, Crowcroft NS, Bontovics E, Deeks SL. Ontario's school-based HPV immunization program: school board assent and parental consent. Can J Public Health 2012; 103(1): 34-39.

43. Dang MT. Let teens provide their own consent for the HPV vaccine. Am J Nurs 2012; 112(6): 11.

44. Hughes C, Jones A, Feemster K \& Fiks A. HPV vaccine decision making in pediatric primary care: a semi-structured interview study. BMC Pediatrics 2011; 11(74): 1-9.

45. Kottow M. Participación informada en clinica e investigación biomédica. Las múltiples facetas de la decisión y el consentimiento informado. UNESCO, Universidad Nacional de Colombia; 2007: 15.

46. Conferencia General de la UNESCO. Declaración Universal sobre Genoma Humano y Derechos Humanos 1997. Ratificada por la Asamblea General de las Naciones Unidas. 1998.

47. Gottvall M, Tydén T, Larsson M, Stenhammar C, Höglund AT. Informed Consent for HPV Vaccination: A Relational Approach. Health Care Anal 2013.

48. Harper DM \& Demars LR. Primary Strategies for HPV Infection and Cervical Cancer Prevention. Clinical obstetrics and gynecology 2014; 57(2): 256-278.

49. Malmqvist E, Helgesson G, Lehtinen J, Natunen K, Lehtinen M. The ethics of implementing human papillomavirus vaccination in developed countries. Medicine, Health Care and Philosophy 2011; 14(1): 20.

50. Alexander NM, Harper DM, Comes JC, Smith MS, Heutinck MA, et al. Intent to Participate in Future Cervical Cancer Screenings Is Lower when Satisfaction with the Decision to Be Vaccinated Is Neutral. Plos One 2014; 9 (6): e98665.

51. Gracia, D. Procedimientos de decisión en ética clínica. Madrid: Triacastela; 2008: 9-102. 
52. Simonsen S. Acceptable Risk in Biomedical Research. European Perspectives. London: Springer Science+Business Media B.V; 2012: 3 .

53. Real W, Alvarado V. Vacunación contra virus papiloma humano: una experiencia chilena en atención primaria. Rev Chilena Infectol 2012; 29(6): 686.

54. Harper D. Conclusions About the Quadrivalent Human Papillomavirus Vaccine Efficacy Based on Alternate Dosing Schedules and Less Than Three Dose Immunogenicity is Inappropriate. J Infect Dis 2014; 210(2): 330-331.

55. Joura EA, et al. A 9-Valent HPV Vaccine against Infection and Intraepithelial Neoplasia in Women. N Engl J Med 2015; 372 (8): 711-723.

56. Torres-Poveda KJ, Cuadra-Hernández SM, Castro-Romero JI, Madrid-Marina V. La política focalizada en el programa de vacunación contra el Virus del Papiloma Humano en México: aspectos éticos. Acta Bioeth 2011; 17(1): 85-94.

57. Steenbeek A, Macdonald N, Downie J, Appleton M, Baylis F. Ill-informed consent? A content analysis of physical risk disclosure in school-based HPV vaccine programs. Public Health Nursing 2012; 29(1): 71-79.

Recibido: 9 de febrero de 2014

Aceptado: 28 de Agosto de 2014 